\title{
3D-Calibration for IMU of the Strapdown Inertial Navigation Systems
}

\author{
V.V. Avrutov ${ }^{1, a}$, M.D. Geraimchuk ${ }^{1}$ and Xing Xiangming ${ }^{2}$ \\ ${ }^{1}$ National Technical University of Ukraine, Kiev Polytechnic Institute, Kiev, Ukraine \\ ${ }^{2}$ Beijing Institute of Control Devices, Beijing, China
}

\begin{abstract}
A new calibration method for Inertial Measurement Unit (IMU) of Strapdown Inertial Navigation Systems was presented. IMU has been composed of accelerometers, gyroscopes and a circuit of signal processing. Normally, a rate transfer test and multi-position tests are using for IMU calibration. The new calibration method is based on whole angle rotation or finite rotation. In fact it's suggested to turn over IMU around three axes simultaneously. In order to solve the equation of calibration, it is necessary to provide an equality of a rank of basic matrix into degree of basic matrix. The results of simulated IMU data presented to demonstrate the performance of the new calibration method.
\end{abstract}

\section{Introduction}

Inertial Measurement Unit (IMU) is a base for developing of Strapdown Inertial Navigation Systems. Each one IMU consist at least three accelerometers and three gyroscopes.

Normally, accelerometers and gyroscopes should go via autonomous testing before they will be assembled to IMU. But it is very important to determinate their features and parameters in whole IMU, because output signals of accelerometers and gyroscopes would be tied to IMU's frame. Therefore IMU calibration is an integral stage of his preparing to work or alignment of the Inertial Navigation System (INS). The calibration is a determination of IMU parameters or his errors for further using or compensation during INS's working.

Usually the IMU calibration is going on by multi-position tests [1-3] for sets of accelerometers, using a precision dividing head. This equipment has a setting accuracy near one second of arc, enables the sensitive axis of an accelerometer rotate with respect to the gravity vector. For calibration of a set of accelerometers it is need to measure output signals, at first to turn the them around $o x$ axis, then to reinstall the set of accelerometers for to measure of output signals by turning around oy axis, and finally to repeat the procedure to measure of output signals by turning around $o z$ axis. Frankly speaking such technology is an artificial case. Actually the vehicle or body turns for whole angle rotation or finite rotation, which is a result of turnings around two or three axes.

For the gyroscopes calibration in IMU by rate transfer tests it is using rate table (precision turntable). During the transfer test the IMU is mounted on the turntable at first with one sensitive axis. For

\footnotetext{
${ }^{a}$ Corresponding author: vyshgorod@gmail.com
} 
example, axis ox is parallel to the axis of rotation of the rate table and rate table is stepped through a series of angular rates $\omega_{x i}$ starting at zero by recording data at each stage. Such test repeated for rotation around axis $o y$ with angular rates $\omega_{y i}$ and, finally, for rotation around axis $o z$ with angular rates $\omega_{z i}, i$ - the number of tests. Need to mark that such method is required a lot of time and at real life the vehicle or body is rotates around the each one of rotation axes.

There are some methods for estimation and compensation of sensors output signal noises. Most known is Kalman filter, used in [4-7]. For estimation of noise features were used Allan variance methods and some time - Wavelet transform [8]. Also, it is well known of using of fuzzy logic techniques [9] and neural networks [10,11].

There is the scalar method of IMU calibration used for gyroscopes and accelerometers [12, 13], based on a scalar reference input motion. For the gyroscopes in the Earth's gravitational field such scalar value is the rotation rate $\Omega$, and for accelerometers - the value of gravity vector $g$.

In this paper was suggested to use a new method of 3D-calibration. The new calibration method based on whole angle rotation. In fact it is suggests to turn over IMU around three axes simultaneously.

\section{Calibration of IMU's set of axial accelerometers}

Output signals of the IMU's axial accelerometers may be expressed according to source [1]:

$$
\left[\begin{array}{l}
U_{a x} \\
U_{a y} \\
U_{a z}
\end{array}\right]=\left[\begin{array}{l}
B_{a x} \\
B_{a y} \\
B_{a z}
\end{array}\right]+\left[\begin{array}{lll}
k_{11} & k_{12} & k_{13} \\
k_{21} & k_{22} & k_{23} \\
k_{31} & k_{32} & k_{33}
\end{array}\right] \cdot\left[\begin{array}{l}
a_{x} \\
a_{y} \\
a_{z}
\end{array}\right]+\left[\begin{array}{l}
n_{a x} \\
n_{a y} \\
n_{a z}
\end{array}\right]
$$

where $B_{a x}, B_{a y}, B_{a z}-g$-independent bias, $a_{x}, a_{y}, a_{z}$-accelerations acting along the $x, y$ and $z$ axes of the sensor respectively, $n_{a x}, n_{a y}, n_{a z}$-zero-mean random biases or output measurements noises, $O x$ $y z$ - body frame, $3 \times 3$ matrix representing the $g$-dependent biases induced by accelerations $a_{x}, a_{y}, a_{z}$, $k_{11}, k_{22}, k_{33}-$ scale-factor coefficients of accelerometers, another one coefficients of the matrix are cross-coupling coefficients.

For reducing noises of output measurements we will average output signals during 100 seconds. Therefore in the future we will ignore of $n_{a x}, n_{a y}, n_{a z}$ value. Let's write the expression (1) for each one accelerometer and for $i$ - position of IMU testing:

$$
\begin{aligned}
& U_{a x i}=B_{a x}+k_{11} a_{x i}+k_{12} a_{y i}+k_{13} a_{z i} ; \\
& U_{a y i}=B_{a y}+k_{21} a_{x i}+k_{22} a_{y i}+k_{23} a_{z i} ; \\
& U_{a z i}=B_{a z}+k_{31} a_{x i}+k_{32} a_{y i}+k_{33} a_{z i} .
\end{aligned}
$$

We will do a set of testing measurements, which are undertaken multi-position tests for turn angles $\alpha, \beta, \gamma$ around three axes simultaneously with exact step of turn (Fig.1). 


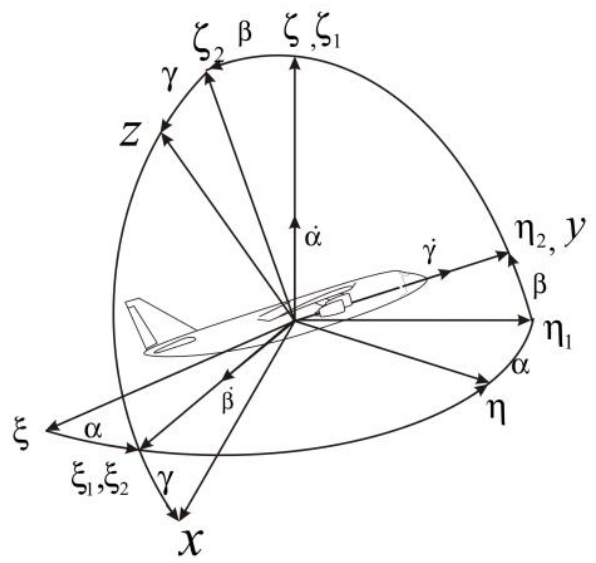

Figure 1. Turn angles $\alpha, \beta, \gamma$ of axial accelerometers

Normally the precision dividing head is using for calibration of accelerometers. But in this case, when IMU is turning around three axes simultaneously or whole angle rotation or finite rotation, the three-axes turntable is used instead a typical precision dividing head.

Let's represent output signal of first accelerometer:

1-st measurement:

$$
U_{a x 1}=B_{a x}+k_{11} a_{x 1}+k_{12} a_{y 1}+k_{13} a_{z 1}
$$

2-nd measurement:

$$
U_{a x 2}=B_{a x}+k_{11} a_{x 2}+k_{12} a_{y 2}+k_{13} a_{z 2}
$$

$n$-st measurement:

$$
U_{a x n}=B_{a x}+k_{11} a_{x n}+k_{12} a_{y n}+k_{13} a_{z n} .
$$

Above set of equations we can represent in matrix form for first, second and third accelerometers:

$$
\begin{aligned}
{\left[\begin{array}{c}
U_{a x 1} \\
U_{a x 2} \\
\vdots \\
U_{a x n}
\end{array}\right]=\left[\begin{array}{cccc}
1 & a_{x 1} & a_{y 1} & a_{z 1} \\
1 & a_{x 2} & a_{y 2} & a_{z 2} \\
\cdot & \cdot & \cdot & \cdot \\
1 & a_{x n} & a_{y n} & a_{z n}
\end{array}\right]\left[\begin{array}{c}
B_{a x} \\
k_{11} \\
k_{12} \\
k_{13}
\end{array}\right] ;\left[\begin{array}{c}
U_{a y 1} \\
U_{a y 2} \\
\vdots \\
U_{a y n}
\end{array}\right]=\left[\begin{array}{cccc}
1 & a_{x 1} & a_{y 1} & a_{z 1} \\
1 & a_{x 2} & a_{y 2} & a_{z 2} \\
\cdot & \cdot & \cdot & \cdot \\
1 & a_{x n} & a_{y n} & a_{z n}
\end{array}\right]\left[\begin{array}{c}
B_{a y} \\
k_{21} \\
k_{22} \\
k_{23}
\end{array}\right] ; } \\
{\left[\begin{array}{c}
U_{a z 1} \\
U_{a z 2} \\
\vdots \\
U_{a z n}
\end{array}\right]=\left[\begin{array}{cccc}
1 & a_{x 1} & a_{y 1} & a_{z 1} \\
1 & a_{x 2} & a_{y 2} & a_{z 2} \\
\cdot & \cdot & \cdot & \cdot \\
1 & a_{x n} & a_{y n} & a_{z n}
\end{array}\right]\left[\begin{array}{c}
B_{a z} \\
k_{31} \\
k_{32} \\
k_{33}
\end{array}\right] }
\end{aligned}
$$

After that let's to combine the received matrix equations to single 'equation of calibration' for IMU's set of accelerometers:

where

$$
\mathbf{U}_{\mathrm{al}}=\mathbf{G}_{\mathrm{n} \times 4} \cdot \mathbf{X}_{1}
$$

$$
\mathbf{U}_{\mathbf{a} 1}=\left[\begin{array}{ccc}
U_{a x 1} & U_{a y 1} & U_{a z 1} \\
U_{a x 2} & U_{a y 2} & U_{a z 2} \\
\cdot & \cdot & \cdot \\
U_{a x n} & U_{a y n} & U_{a z n}
\end{array}\right], \quad \mathbf{G}_{\mathbf{n} \times 4}=\left[\begin{array}{cccc}
1 & a_{x 1} & a_{y 1} & a_{z 1} \\
1 & a_{x 2} & a_{y 2} & a_{z 2} \\
\cdot & \cdot & \cdot & \cdot \\
1 & a_{x n} & a_{y n} & a_{z n}
\end{array}\right], \quad \mathbf{X}_{1}=\left[\begin{array}{ccc}
B_{a x} & B_{a y} & B_{a x} \\
k_{11} & k_{21} & k_{31} \\
k_{12} & k_{22} & k_{32} \\
k_{13} & k_{23} & k_{33}
\end{array}\right] .
$$

We will solve the last matrix equation by least-squares method:

$$
\hat{\mathbf{X}}_{1}=\left(\mathbf{G}_{\mathrm{n} \times 4}^{\mathrm{T}} \mathbf{G}_{\mathrm{n} \times 4}\right)^{-1} \mathbf{G}_{\mathrm{n} \times 4}^{\mathrm{T}} \mathbf{U}_{\mathrm{a} 1} \text {. }
$$


Here symbol 'T, is mean a transposed matrix.

Sample 1. We will consider the IMU's accelerometers with the below followings nominal parameters:

$$
\begin{aligned}
& B_{a x}=B_{a y}=B_{a z}=2.5 \mathrm{~V} / g ; k_{11}=k_{22}=k_{33}=1.0 \mathrm{~V} / \mathrm{g} ; \\
& k_{12}=0.01 \mathrm{~V} / g, k_{13}=-0.01 \mathrm{~V} / \mathrm{g}, k_{21}=-0.01 \mathrm{~V} / \mathrm{g}, \\
& k_{23}=0.01 \mathrm{~V} / g, k_{31}=0.01 \mathrm{~V} / g, k_{32}=-0.01 \mathrm{~V} / \mathrm{g},
\end{aligned}
$$

Body's turns with angles $\alpha, \beta, \gamma$ wich will change from 0 to 400 degree with the same step of 10 degree (the number of positions is 40 ).

The calculated values of output signals (2) for matrix $\mathbf{G}_{\mathbf{n} \times \mathbf{4}}$ are shown on Fig. 2.

After calculations according to (3), we will have:

$$
\hat{\mathbf{X}}_{\mathbf{1}}=\left[\begin{array}{ccc}
2.5 & 2.5 & 2.5 \\
1.0 & -0.01 & 0.009999 \\
0.01 & 1.0 & -0.009999 \\
-0,009999 & 0.01 & 1.0
\end{array}\right] \text {. }
$$

Thus, as a result of measuring (2) and calculations (3) it is succeeded to get estimations of the biases $\hat{\mathbf{B}}_{\mathbf{a}}$, scale factors and cross-coupling coefficients - elements of matrix $3 \times 3$.
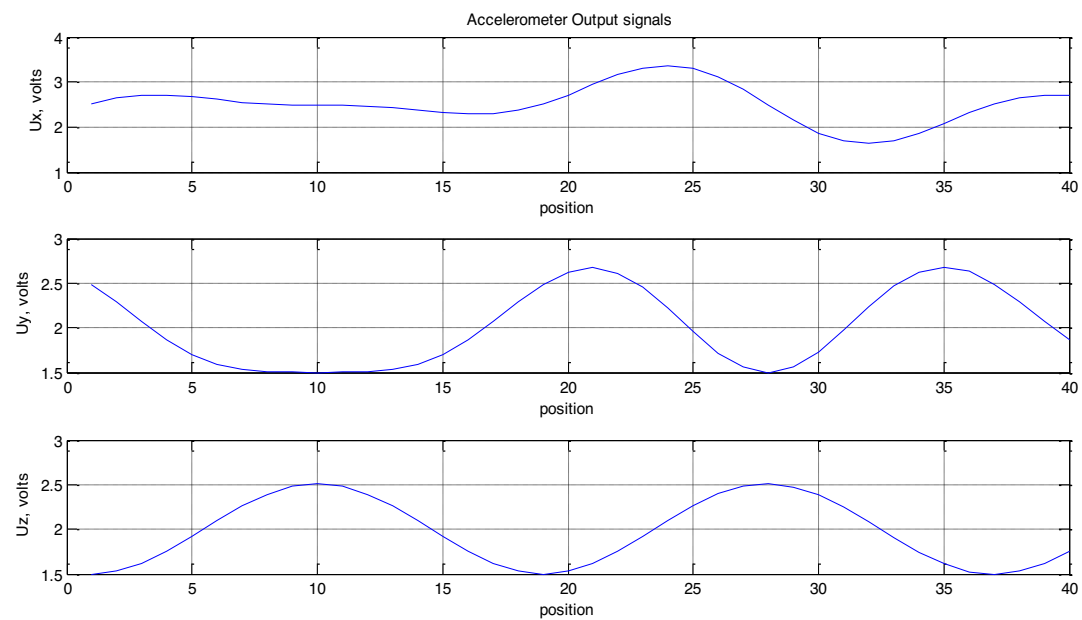

Figure 2. Averaging output signals of axial accelerometers

\section{Calibration of IMU's set of gyroscopes}

Output signals of the IMU's gyroscopes may be expressed according to source [1]:

$$
\left[\begin{array}{c}
U_{\omega x} \\
U_{\omega y} \\
U_{\omega z}
\end{array}\right]=\left[\begin{array}{c}
B_{\omega x}^{*} \\
B_{\omega y}^{*} \\
B_{\omega z}^{*}
\end{array}\right]+\left[\begin{array}{lll}
n_{11} & n_{12} & n_{13} \\
n_{21} & n_{22} & n_{23} \\
n_{31} & n_{32} & n_{33}
\end{array}\right] \cdot\left[\begin{array}{c}
\omega_{x} \\
\omega_{y} \\
\omega_{z}
\end{array}\right]+\left[\begin{array}{c}
n_{\omega x} \\
n_{\omega y} \\
n_{\omega z}
\end{array}\right] .
$$

Here $B_{\omega x}^{*}, B_{\omega y}^{*}, B_{\omega z}^{*}$ - biases of each one gyroscopes, which may be depends from $g$ and $\mathrm{g}^{2}-$ drifts, $\omega_{x}, \omega_{y}, \omega_{z}$ - turn rates, acting along the $x, y$ and $z$ axes of the sensor respectively, $n_{\omega x}, n_{\omega y}, n_{\omega z}$ - zeromean random biases or output measurements noises, $3 \times 3$ matrix representing the biases induced by $\omega_{x}$, $\omega_{y}, \omega_{z}, n_{11}, n_{22}, n_{33}$ - scale-factor coefficients of gyroscopes, another one coefficients of the matrix are cross-coupling coefficients. 
It's known [1], that $g$ and $\mathrm{g}^{2}$ dependent biases are usually insignificant in optical sensors such as fiber optic and ring laser gyroscopes.

Consider a calibration such optical sensors when IMU turns around three axes simultaneously. When the set of $n$-measurements will be done with turn rates $\omega_{x i}, \omega_{y i}, \omega_{z i}$ and after averaging of output signals during 100 seconds we will have a new matrix 'equation of calibration':

$$
\mathbf{U}_{\omega 3}=\omega_{n \times 4} \cdot X_{3} \text {, }
$$

where

$$
\mathbf{U}_{\omega 3}=\left[\begin{array}{ccc}
U_{\omega x 1} & U_{\omega y 1} & U_{\omega z 1} \\
U_{\omega x 2} & U_{\omega y 2} & U_{\omega z 2} \\
\cdot & \cdot & \cdot \\
U_{\omega x n} & U_{\omega y n} & U_{\omega z n}
\end{array}\right], \mathbf{X}_{3}=\left[\begin{array}{ccc}
B_{\omega x}^{*} & B_{\omega y}^{*} & B_{\omega z}^{*} \\
n_{11} & n_{21} & n_{31} \\
n_{12} & n_{22} & n_{32} \\
n_{13} & n_{23} & n_{33}
\end{array}\right], \boldsymbol{\omega}_{\mathbf{n} \times 4}=\left[\begin{array}{cccc}
1 & \omega_{x 1} & \omega_{y 1} & \omega_{z 1} \\
1 & \omega_{x 2} & \omega_{y 2} & \omega_{z 2} \\
\cdot & \cdot & \cdot \\
1 & \omega_{x n} & \omega_{y n} & \omega_{z n}
\end{array}\right] .
$$

On the face of it the structure of the equation (5) is very similar to equation (2). The difference is that matrix $\boldsymbol{\omega}_{\mathbf{n} \times \mathbf{4}}$ consists from turn rates $\omega_{x i}, \omega_{y i}, \omega_{z i}$ and matrix $\mathbf{G}_{\mathbf{n} \times \mathbf{4}}$ - from accelerations $a_{x i}, a_{y i}, a_{z i}$. However if we have ordered turn series without any limitations for calibration of IMU's set of accelerometers, otherwise we should provide a below following rule:

An equation of calibration will have a decision if the rank of coefficient matrix $\boldsymbol{\omega}_{\mathbf{n} \times \mathbf{m}}$ is equal to number of columns of the same matrix or by other words $\operatorname{rank} \boldsymbol{\omega}_{\mathbf{n} \times \mathbf{m}}=\mathbf{m}$.

The above rule is based on Rouche-Capelli or Kronecker-Capelli theorem [14]. A system of linear equations with $n$ variables has a solution if and only if the rank of its coefficient matrix $\boldsymbol{\omega}_{\mathbf{n} \times \mathbf{4}}$ is equal to the rank of its augmented matrix $\left[\boldsymbol{\omega}_{\mathbf{n} \times \mathbf{4}}, \mathbf{U}_{\boldsymbol{\omega} 3}\right]$ :

$$
\operatorname{rank} \boldsymbol{\omega}_{\mathbf{n} \times \mathbf{4}}=\operatorname{rank}\left[\boldsymbol{\omega}_{\mathbf{n} \times \mathbf{4}}, \mathbf{U}_{\mathbf{\omega} 3}\right] \text {. }
$$

This condition could be provided, for example due nonlinear depending of last columns of matrix $\omega_{n \times 4}$.

Solving the last matrix equation (5) by least-squares method:

$$
\hat{\mathbf{X}}_{3}=\left(\omega_{n \times 4}^{\mathrm{T}} \omega_{n \times 4}\right)^{-1} \omega_{n \times 4}^{\mathrm{T}} \mathbf{U}_{\omega 3} .
$$

Sample 3. Consider the IMU's gyroscopes with the below followings nominal parameters:

$B^{*}{ }_{\omega x}=B^{*}{ }_{\omega y}=B^{*}{ }_{\omega z}=2.0 \mathrm{~V}$;

$n_{11}=0.01 \mathrm{~V} /(\mathrm{d} / \mathrm{s}) ; \quad n_{12}=0.01 \mathrm{~V} /(\mathrm{d} / \mathrm{s}) ; n_{13}=-0.02 \mathrm{~V} /(\mathrm{d} / \mathrm{s})$;

$n_{21}=-0.01 \mathrm{~V} /(\mathrm{d} / \mathrm{s}) ; n_{22}=0.1 \mathrm{~V} /(\mathrm{d} / \mathrm{s}) ; \quad n_{23}=0.01 \mathrm{~V} /(\mathrm{d} / \mathrm{s}) ;$

$n_{31}=0.03 \mathrm{~V} /(\mathrm{d} / \mathrm{s}) ; n_{32}=-0.01 \mathrm{~V} /(\mathrm{d} / \mathrm{s}) ; n_{33}=0.1 \mathrm{~V} /(\mathrm{d} / \mathrm{s})$;

To provide the rank of matrix $\boldsymbol{\omega}_{\mathbf{n} \times \mathbf{4}}$ to order of matrix:

$$
\operatorname{rank} \boldsymbol{\omega}_{\mathrm{n} \times \mathbf{4}}=4 \text {, }
$$

we will arrange the projections of angular rate of turntable in below following series:

$$
\omega_{x i}=\omega_{i}, \quad \omega_{y i}=\omega_{i}^{1 / 2}, \quad \omega_{z i}=\omega_{i}^{1 / 3} .
$$

We will change the angular rate $\omega_{i}$ from 0 to 100 degree/sec with the same step of 10 degree/sec. The number of tests is 10 .

Solving the equation of calibration according to (6) by least-squares method we will have 


$$
\hat{\mathbf{X}}_{3}=\left[\begin{array}{ccc}
2.0 & 2.0 & 2.0 \\
1.0 & -0.01 & 0.03 \\
0.01 & 1.0 & -0.01 \\
-0.02 & 0.01 & 1.0
\end{array}\right] \text {. }
$$

Thus, as a result of measuring (5) and calculations (6) it is succeeded to get estimations of the gyro's biases and elements of the matrix $3 \times 3$.

\section{Conclusions}

A new calibration method for IMU of Strapdown Inertial Navigation Systems was suggested. The new calibration method is based on whole angle rotation or finite rotation. In fact it is suggested to turn over Inertial Measurement Unit around three axes simultaneously. In order to solve the equation of calibration, it is necessary to provide an equality of a rank of basic matrix to degree of basic matrix. The results of simulated IMU data are presented to demonstrate the performance of the new calibration method.

\section{References}

1. Titterton D. H. and Weston J. L. Strapdown Inertial Navigation Technology. - IEE Radar, Sonar, Navigation and Avionics Series 17, 2004. - pp. 558.

2. Nebot E., Durrant-Whyte H., Initial Calibration and Alignment of Low Cost Inertial Navigation Units for Land Vehicle Applications. - Journal of Robotics Systems, Vol. 16, No. 2, February 1999, pp. 81-92.

3. Artese G., Trecroci A. Calibration of a low cost MEMS INS sensor for an integrated navigation system. - The International Archives of the Photogrammetry, Remote Sensing and Spatial Information Sciences. Vol. XXXVII. Part B5. Beijing 2008, pp. 877-882.

4. Grewal M. S., Henderson, V. D., Miysako R. S. Application of Kalman filtering to the calibration and alignment of inertial navigation systems. IEEE Transactions on Automatic Control, 1991, vol. 36, pp. 3-13.

5. Hide C., Moore T., Smith M. Adaptive Kalman filtering for low-cost INS/GPS. The Journal of Navigation, 2003, vol. 56, pp. 143-152.

6. Nikbakht N., Mazlom M., and Khayatian A. Evaluation of solid-state accelerometer for positioning of vehicle. Proceeding of the IEEE International Conference on Industrial Technology, Hong Kong, 2005, pp. 729-733.

7. Pang G., Liu, H. Evaluation of a low-cost MEMS accelerometer for distance measurement. The Journal of Intelligent and Robotic Systems, 2001, vol. 30, pp. 249-265.

8. Sheng-Chih Shen, Chia-Jung Chen, Hsin-Jung Huang. A new calibration method for low cost MEMS inertial sensor module. Journal of Marine Science and Technology, 2010, vol. 18, No. 6, pp. 819-824.

9. Gaysse, J. A low cost absolute position calculation system. Proceeding of SICE-ICASE International Joint Conference, Busan, Korea, 2006, pp. 5658-5661.

10. El-Rabbany A., El-Diasty, M. An efficient neural modal for denoising of MEMS-based inertial data. The Journal of Navigation, 2004, vol. 57, pp. 407-415.

11. Wang H., Tian W. Modeling the random drift of micro-machined gyroscope with neural network. Neural Processing Letters, 2005, vol. 22, pp. 235-247.

12. Avrutov V. On Scalar Calibration of an Inertial Measurement Unit / V. Avrutov, S. Golovach, T. Mazepa // 19-th St. Petersburg International Conference on Integrated Navigation Systems. St. Petersburg, Russia, 2012. - State Research Center (CSRI) Elektropribor, 2012, pp.117-121. 
13. Avrutov V. Scalar Diagnostics of the Inertial Measurement Unit// I.J. Intelligent Systems and Applications, 2015, vol.11, pp.1-9.

14. Bugrov Y.S., Nikolsky S.M. Higher mathematics. Elements of linear algebra and analytic geometry. - Moscow: 'Nauka', 1984. - pp.192 (In Russian). 